\title{
Level of Professionalism among Nurses Working in Azad Kashmir
}

\author{
Shahnaz Amir ${ }^{1 *}$, Dr. Mansoor Ghani ${ }^{2}$, Nazia Ahmad Buksh ${ }^{3}$, Tanzeel-u-Rehman ${ }^{4}$, Asma Gul $^{5}$
}

${ }^{1,3,5}$ Student, University of Health Sciences, Lahore, Pakistan

${ }^{2}$ Head of Department, University of Health Sciences, Lahore, Pakistan

${ }^{4}$ Nursing Instructor, Regional Training Institute, Lahore, Population Welfare Department Punjab, Pakistan

\author{
DOI: $\underline{10.36348 / \text { sjnhc.2020.v03i06.004 }}$ \\ | Received: 16.06.2020 | Accepted: 23.06.2020 | Published: 30.06.2020 \\ *Corresponding author: Shahnaz Amir
}

\section{Abstract}

Objective: To assess the level of professionalism among nurses working in Azad Kashmir also to identify nurses' perceived strategies to enhance professionalism among nurses. Study Design: A descriptive cross sectional study. Place and Duration: Twelve months, from April 2017, five tertiary care hospitals of Azad Kashmir. Methods: The study included nurses who had been providing care in five tertiary care hospitals of Azad Kashmir since 3 years. (N=150). Data was collecting by using a self-structured questionnaire. Results: Research findings revealed that by using arbitrary scale $50(33.3 \%)$ out of 150 participants got more than $60 \%$ score on Likert scale; they had high level of professionalism. While $15(10 \%)$ respondent got more than $80 \%$ score which denote exemplary level of professionalism. Likewise, 24(16\%) nurses obtained $40 \%$ and less than $40 \%$ score which expresses low level of professionalism; moreover 61(40.6\%) nurses demonstrated average professional level. Conclusion: Findings of the present study display that generally professionalism among nurses is at average level. Although level of professionalism in areas of Ethical practice and Responsibility and Accountability is relatively high. Some areas showed very low level of professionalism among nurses. These are Research Participation and Utilization, Autonomy. This study advocate for less stressful and less oppressed working environment coupled with opportunity to engaging in continue education for enhancing professionalism.

Keywords: Professionalism, Ethical Practice, Self-Development, Autonomy.

Copyright @ 2020: This is an open-access article distributed under the terms of the Creative Commons Attribution license which permits unrestricted use, distribution, and reproduction in any medium for non-commercial use (NonCommercial, or CC-BY-NC) provided the original author and source are credited.

\section{INTRODUCTION}

Professionalism is defined as a set of merits involves the conscious application of the principles of Excellency, humanism, accountability and altruism that based on admirable clinical competence, interpersonal relationships, effective communication skills and ethical standards [1].

The pace of change is unrelenting, due to ever changing health care system nursing profession encounters enormous ethical and philosophical challenges. These challenges schemed the role of the nurse more complicated, sophisticated and highly professional rather than just experienced [2] and is linked to divers' responsibilities [3].

Nursing professionalism is a inductee to provision of compassionate care, adherence to strong ethical values, continues self-development, accountability and responsibility for discerning practice, representing the spirit of Collegiality and collaboration [2]. Compassion, care and patient centeredness are central to nursing profession [2]. Trusting and credulous relationship between nurse and patient is the key feature of nursing profession. From this point of view, individuals, families and communities expect nurses to show certain professional attributes while providing nursing care. These attributes may comprise of broad knowledge skills and competencies, accountability, integrity, altruism, effective communication, empathy and courtesy [4].

Studies suggested that high quality of care, patient satisfaction, trust on nurses and compliance to treatment plans are strongly associated with health professional's behaviors that evident professionalism as having a caring attitude, listening, providing sufficient relevant information, being efficient and competent, and have aptitude of healthy teamwork [5]. Compromised professionalism impact negatively not only on individual, but also on the institution and quality of the services provided as well [6].

High level of professionalism has association with sound incredible nursing performance, personal and professional autonomy, critical thinking, clinical reasoning, empowerment and ability to reflect on 
practice. Nurses with a higher degree of professionalism have been reported to have better levels of education and training, active participation in research activities and well-motivated for application of evidence-based practice. On the other hand, low levels of professionalism may result in decreased productivity, patient dissatisfaction, low morale, attrition and turnover [4].

Demonstration of professionalism at work place increase recognition and professional dignity of nurses. Recognition and appreciation of the professional dignity of nurses plays a pivotal role in improving their clinical performance, fostering relationship with health care team, increased job satisfaction and productivity. If nurse's professional role remains unnoticed and unrecognized by other member of health care team, they will not be able to provide effective care [7]. Professionalism is an important characteristic of careers that highlight values and commitment in service to the society. Professionalism is a multifaceted concept that provides guidance for nurses to ameliorate personally and professionally. It is imperative to correct the image of nursing and enhance the standard of the profession. Through self-regulation, adherence to social and ethical values, the maintenance of high ethical standards and commitment to continue professional development, nurses can achieve the goal of positive image and high professional standard [8]. Negative public image can drastically affect future of nursing in many ways; it distorts the public concept of nursing and strengthens negativity and myths about nursing profession. Such image may decrease the probability of intelligent and bright people to joining nursing as a profession. Negative image may lead to scarcity of resources allocation by policymaker for nursing profession [9].

Awareness and development of professionalism is continuing and life long process. Initiatives on local, national and international level are required to support and enhance professionalism in nursing. Nurses need to improve their attitude, knowledge and skills, whereas institute takes responsibility of providing facilities for learning and continue nursing education [2].

Individually the levels of professionalism of nurse may affect clinical practice, bedside monitoring and patient safety [10]. Development of professionalism is essential for quality improvement in patient care. Quality of patient care is highly depending on performance and behavior of nurses and physician.

\section{METHODOLOGY}

Study Design: Descriptive Cross-sectional design

\section{Setting}

This study was carried out at Institute of Nursing, University of health science, Lahore Pakistan, and data was collected from five selected tertiary care hospitals of Azad Kashmir including, Sheikh Khalifa Bin Zaid Al-Nahyan Hospital Rawalakot, Abass Institute of Medical Scinces (AIMS) Muzaffarabad, Sheikh Khalifa Bin Zaid Al-Nahyan Hospital (SKBZAN) Hospital Muzaffarabad, Divisional Headquarter Teaching Hospital Mirpur and New City Teaching Hospital Mirpur.

Subjects: The study included nurses who have more than 3 year clinical experience. $(\mathrm{N}=150)$.

Inclusion Criteria: Registered nurses having experience more than two years.

Exclusion Criteria: Nurses working on temporary basis.

\section{Ethical Considerations}

Ethical approval was taken from Ethical Review Committee of University of Health Sciences Lahore, Pakistan. Written permission was obtained from deans/medical directors of respective study hospitals. Written consent describing the purpose and benefits of the study was taken from study participants.

\section{Tools of Data Collection}

Tool for this study was developed in three sections.

Section-1: consists of socio demographic characteristics of participants including age, gender, marital status, level of education, designation, Year of experience and Registration with professional organizations.

Section-2: Comprised of 43 questions about level of professionalism and further divided into eight areas according to basic characteristics of professionalism. These areas are; (A)Professional Responsibility \& Accountability (B)Knowledge Based Practice), (C)Client- Focused Provision of Care, (D)Ethical Practice, (E)Communication \& Interpersonal Relationships, (F)Autonomy, (G)Research participation $\&$ utilization and (H)Self-Regulation \& continue education. This section consists of 43 questions. A five points likert scale from Never to Always $(1=$ Never, $2=$ Rarely, 3=Unsure, 4=Often and 5= Always) was used for recording the responses.

Following indicators were used to evaluate the level of professionalism

Low level $<40 \%$

Average (need improvement $41 \%-60 \%$

High level $>60 \%$

Exemplary $>80 \%$

Section 3: Comprises of 15 questions about the strategies to enhance professionalism. Responses was collected by using four points Likert scale not important to extremely important ( $1=$ Not important, $2=$ somewhat important, $3=$ Important, $4=$ extremely important). 


\section{Validity and reliability of Data Collection Tools}

In current study, internal consistency reliability of self-constructed questionnaire was tested through pilot study by applying Cronbach's Alpha through SPSS IBM version 20. It was calculated as with internal consistency of 0.913 for section 2 (professionalism) and 0.871 for section 3 , which indicated that the instrument was reliable for data collection.

\section{Pilot Study}

Before embarking on the full study, selfconstructed questionnaire was pre-tested. A pilot study was carried out on $27(20 \%)$ staff nurses to test the feasibility of study design, impartiality and applicability of data collection tool. Participants were selected according to their voluntary consent on basis of nonprobability sampling technique. Selected participants were not included in actual study.

\section{Data Collection Procedure}

All the participants were included in the study on the bases of fulfilment of inclusion criteria. Data was collected from 150 nurses working in tertiary care hospital of Azad Kashmir after the approval of concerned authorities. Written Informed consent was obtained and purpose of study was explained. Proper explanation and instructions were given to the participants regarding responses to questions asked in questionnaire. Confidentiality and privacy ensured and maintained. The data was collected by self-administered questionnaire. After getting response from subjects, questionnaire were collected and considered as final without any manipulation. Incomplete questionnaire were not included in study. Obtained data were converted into numeric form, and the average of two observations was calculated.

\section{Data Analysis}

The response rate was $100 \%$. Data was processed and analysed through SPSS software version IBM 20. Descriptive statistics (mean, standard deviation, frequencies and percentages) were used to describe the results.

\section{RESULTS}

\section{Section 1}

Table-1 describes demographic characteristics of participant nurses which include gender, qualification, experience, designation, registration with professional organization and foreign working experience.
Table-1: Frequency distribution of Demographic Characteristics of Studied Sample $(\mathbf{N}=150)$

\begin{tabular}{|c|c|c|}
\hline & Frequency & Percentage \\
\hline \multicolumn{3}{|l|}{ Gender } \\
\hline Female & 150 & 100.0 \\
\hline \multicolumn{3}{|l|}{ Qualification } \\
\hline Diploma & 85 & 56.7 \\
\hline Post RN & 21 & 14.0 \\
\hline Specialization & 38 & 25.3 \\
\hline MSc Nursing & 06 & 4.0 \\
\hline Total & 150 & 100.0 \\
\hline \multicolumn{3}{|l|}{ Designation } \\
\hline Bed side nurse & 120 & 80.0 \\
\hline Head Nurse & 19 & 12.7 \\
\hline Nursing Instructor & 11 & 7.3 \\
\hline Total & 150 & 100.0 \\
\hline \multicolumn{3}{|l|}{ Experience } \\
\hline $2-6$ years & 56 & 37.3 \\
\hline $7-11$ years & 35 & 23.3 \\
\hline 12-16 years & 19 & 12.7 \\
\hline 17-20 years & 21 & 14.0 \\
\hline$>20$ years & 19 & 12.7 \\
\hline Total & 150 & 100.0 \\
\hline \multicolumn{3}{|c|}{ Registration with professional organization } \\
\hline $\mathrm{PNC}$ & 150 & 100.0 \\
\hline Total & 150 & 100.0 \\
\hline \multicolumn{3}{|c|}{ Foreign working experience of Participants } \\
\hline No. & 150 & 100.0 \\
\hline Total & 150 & 100.0 \\
\hline
\end{tabular}

Demographic Characteristics of Studied Sample $(N=150)$

\section{Section II}

\section{Level of Professionalism among nurses}

Level of professionalism among nurses of tertiary care hospital of Azad Kashmir was at average level. Sixty one $(40.6 \%)$ out of 150 participants got the scores $40 \%$ to $50 \%$ on likert scale which represent average level of professionalism. Similarly 24(16\%) respondent got $\geq 40 \%$ score which denoted low level of professionalism. While exemplary level seen only in $15(10 \%)$ respondent who got more than $80 \%$ score on likert scale (Figure-1).

Table-2 illustrates frequency distribution of Level of professionalism according to characteristics of professionalism among studied sample. 
Table-2: Frequency distribution of Level of professionalism according to characteristics of professionalism among studied sample $(\mathrm{N}=\mathbf{1 5 0})$

\begin{tabular}{|c|c|c|}
\hline & Frequency & Percentage \\
\hline \multicolumn{3}{|c|}{ Professional Responsibility \& Accountability } \\
\hline Low level & 8 & 5.3 \\
\hline Average & 70 & 46.7 \\
\hline High level & 40 & 26.7 \\
\hline Exemplary & 32 & 21.3 \\
\hline Total & 150 & 100.0 \\
\hline \multicolumn{3}{|c|}{ Knowledge Based Practice } \\
\hline Low level & 35 & 23.3 \\
\hline Average & 81 & 54.0 \\
\hline High level & 21 & 14.0 \\
\hline Exemplary & 13 & 8.7 \\
\hline Total & 150 & 100.0 \\
\hline \multicolumn{3}{|c|}{ Client Focus Provision of Care: } \\
\hline Low level & 30 & 20.0 \\
\hline Average & 59 & 39.3 \\
\hline High level & 40 & 26.7 \\
\hline Exemplary & 21 & 14.0 \\
\hline Total & 150 & 100.0 \\
\hline \multicolumn{3}{|c|}{ Ethical Practice } \\
\hline Low level & 6 & 4.0 \\
\hline Average & 34 & 22 \\
\hline High level & 27 & 18 \\
\hline Exemplary & 83 & 55.3 \\
\hline Total & 150 & 100.0 \\
\hline \multicolumn{3}{|c|}{ Communication and inter-personal relationshi } \\
\hline Low level & 16 & 10.7 \\
\hline Average & 87 & 58.0 \\
\hline High level & 26 & 17.3 \\
\hline Exemplary & 21 & 14.0 \\
\hline Total & 150 & 100.0 \\
\hline \multicolumn{3}{|l|}{ Autonomy } \\
\hline Low level & 99 & 66.0 \\
\hline Average & 42 & 28.0 \\
\hline High level & 9 & 6.0 \\
\hline Total & 150 & 100.0 \\
\hline \multicolumn{3}{|c|}{ Research participation \& utilization } \\
\hline Low level & 106 & 70.7 \\
\hline Average & 36 & 24.0 \\
\hline High level & 8 & 5.3 \\
\hline Total & 150 & 100.0 \\
\hline \multicolumn{3}{|c|}{ Self-Regulation \& continue education } \\
\hline Low level & 20 & 13.3 \\
\hline Average & 75 & 50.0 \\
\hline High level & 32 & 21.3 \\
\hline Exemplary & 23 & 15.3 \\
\hline Total & 150 & 100.0 \\
\hline
\end{tabular}

Professionalism according to characteristics of professionalism among studied sample (N=150)

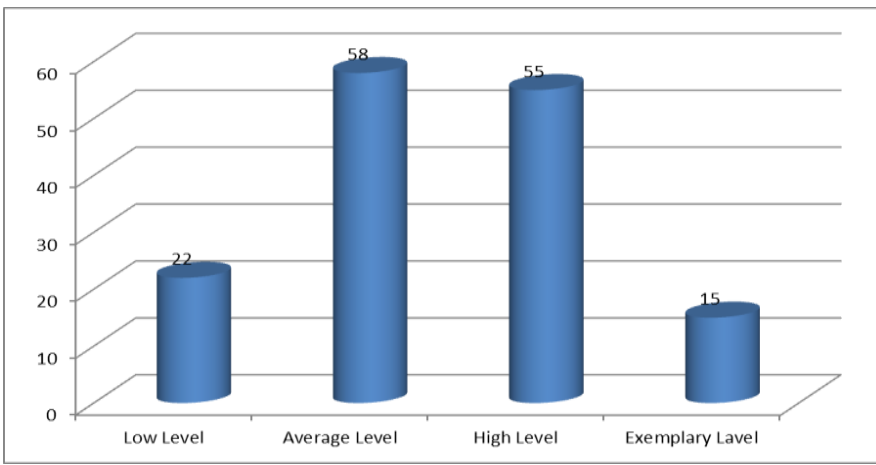

Fig-1: Frequency distribution of Level of professionalism among studied sample $(\mathrm{N}=150)$ 


\section{Status of Professionalism}

Considering about Level of professionalism according to different characteristics of nursing professionalism it was observed that ethical practice was highest area among nurses with mean value of 77.5 \pm 19.16 SD. Similarly, domain of self-regulation and continue education was on second number with mean of $76.0 \pm 22.0$ SD.

On the other hand, area of autonomy and research participation and utilization was lowest scored area among nurses with mean value $37.8 \pm 15.2 \mathrm{SD}$ and $39.5 \pm 15 \mathrm{SD}$ respectively.

Discussing about knowledge-based practice it was seen that respondent nurses demonstrated average

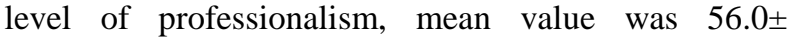
26.2SD. Similar findings were seen in area of communication which mean value was $64.2 \pm 20.1 \mathrm{SD}$. Furthermore, mean value of professionalism in area of Responsibility and Accountability was $75.2 \pm 22.1 \mathrm{SD}$ and $69.2 \pm 22.1 \mathrm{SD}$ was observed for client focus provision of care (Figure-2).

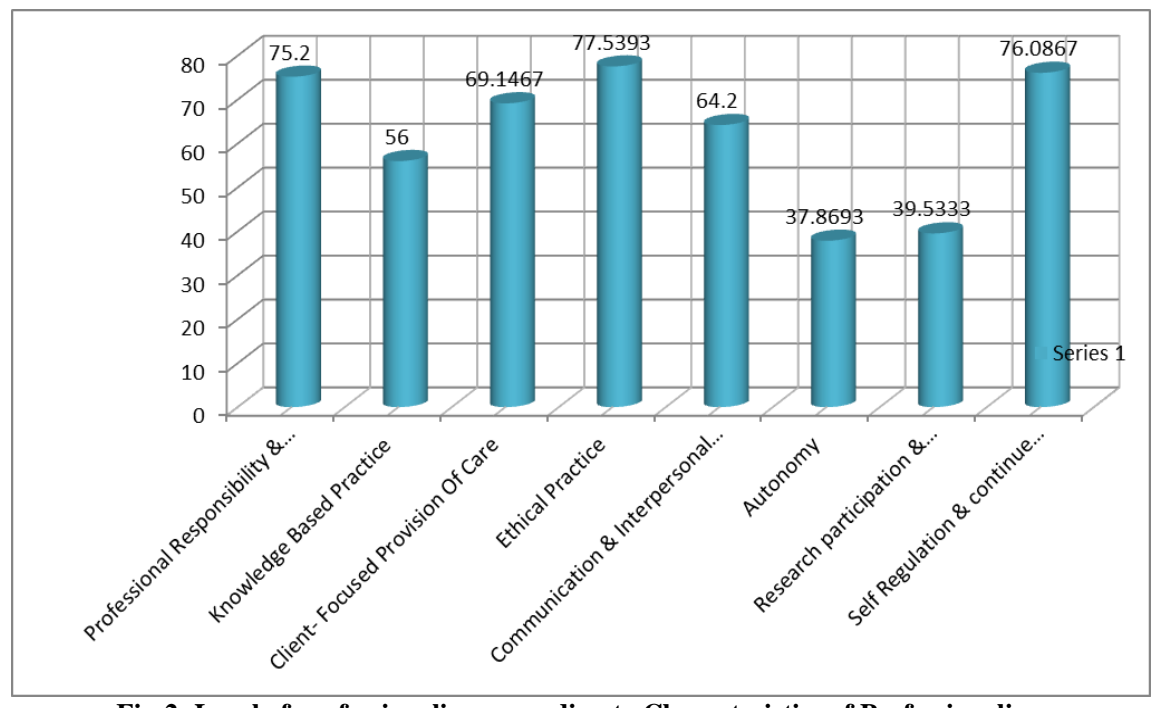

Fig-2: Level of professionalism according to Characteristics of Professionalism

\section{Characteristics of professionalism}

Section-III

Section-3 consists of 14 strategies. Respondent were asked to rate them according to their importance for enhancing professionalism among nurse. Continue education in form of workshops, seminar and conference was highest rated strategy among these; nurses perceived it extremely important and play key role in development of professionalism.Mean score was $3.83+0.38 \mathrm{SD}$, similarly subjects placed continue education in the form of $\mathrm{BSc}$ and MSc nursing on second priority with mean score of $3.65+0.518 \mathrm{SD}$ (Figure-3).

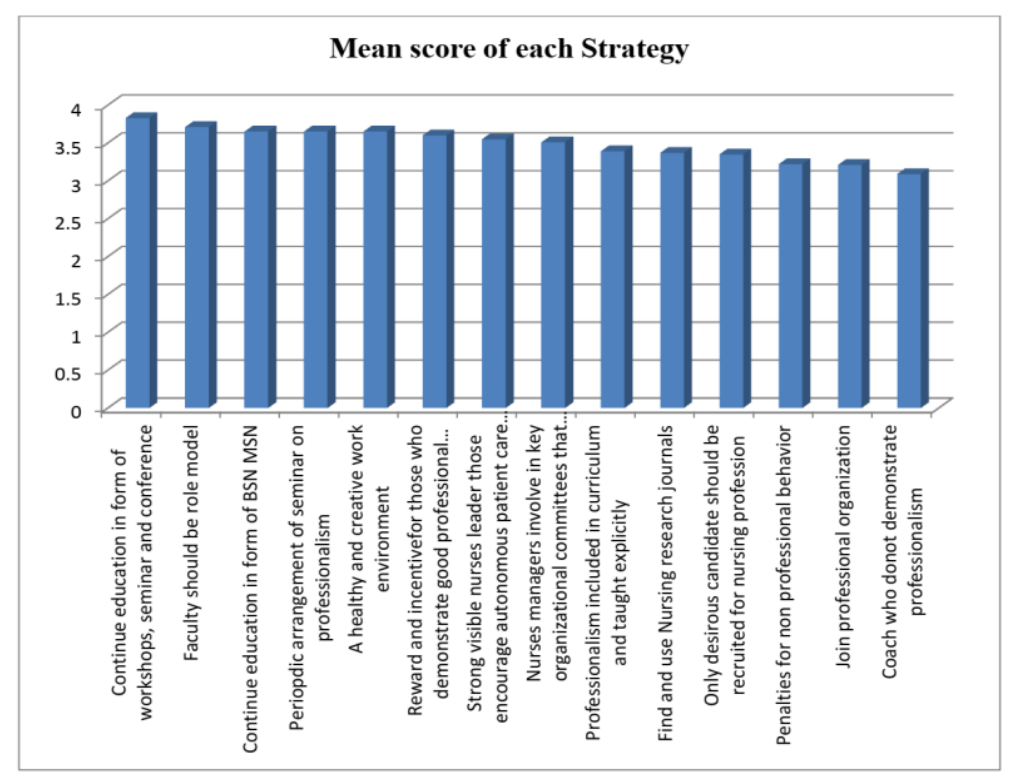

Fig-3: Mean score for each strategy 


\section{DISCUSSION}

Findings of this study revealed that overall level of professionalism among studied sample was at average level. Sixty one $(40.6 \%)$ out of 150 participants got more than $40 \%$ to $50 \%$ score on likert scale; represent average level of professionalism. Similarly $24(16 \%)$ respondent got $\geq 40 \%$ score which denoted low level of professionalism. While only $15(10 \%)$ respondent got more than $80 \%$ score which denote exemplary level of professionalism.

Results of this study were supported by results of study conducted by [11] and the result of a study conducted in North Ethiopia by [12]. Findings of this study were opposed by study conducted by [13] They proposed that nurses display high level of professionalism. Difference in nursing practice setting and nursing education are among the reasons of this incongruence.

According to different domains of professionalism it was seen that nurses got the lowest score in domain of autonomy. Mean score was 37.8 +15.2 SD. Similar findings were reported by $[14,12]$ who reported that autonomy was the lowest scored area among the domains of professionalism. Extremely hierarchic and strict administrative structure along with regulatory law for nursing practice may be responsible for insufficient autonomy among nurses

Another lowest scored area of professionalism was Research participation and utilization. Mean score for Research participation and utilization was $39.5+15$ SD. These findings were in congruence with findings of study conducted in Turkey by [15] and study by Karadağ et al., [11].

These results were incongruent with the findings of a study conducted in japan by [16] which showed that nurses obtained highest score in the area of research and publications. Nurses have little or no opportunity for participation in research because of absence of research culture and little trend of evidence based practice.

Ethical practice was the domain in which nurses got highest score among all domains of professionalism with the mean score of 77.5+19SD. The finding is in concordance with [17]. A Gallup surveys was conducted on 2006, 2010, 2017 and 2018 [18]. According to Gallup survey 2010 public rated the nursing profession as highest regarding honesty and ethical practice [17]. The findings of [14] also agree with the findings of current study.

Concerning about self-regulation and continue education it was seen that $74 \%$ nurses represent higher level of professionalism with mean score 76.0+22.0 SD. These findings were in compliance with that of $[18,19]$.

\section{CONCLUSION}

Findings of the present study showed that generally professionalism among nurses working in tertiary care hospital of Azad Kashmir is average. Although level of professionalism in some sub areas like ethical practice and responsibility and Accountability is relatively raised. Some areas showed very low level of professionalism among nurses like research participation and utilization and autonomy. Due to overburden nurses have lack of creative working environment and research culture. This study advocate for less stressful and less oppressed working environment coupled with opportunity to engaging in continue education for enhancing professionalism.

\section{REFERENCES}

1. Jakovljević, M., \& Ostojić, L. (2013). Professionalism in contemporary medicine: If it is an important academic issue, then surely it is a "hot" issue as well. Medicina, 1, 6-17.

2. Ghadirian, F., Salsali, M., \& Cheraghi, M. A. (2014). Nursing professionalism: An evolutionary concept analysis. Iranian journal of nursing and midwifery research, 19, 1.

3. Mrayyan, M. T. (2004). Nurses' autonomy: influence of nurse managers' actions. Journal of advanced nursing, 45, 326-336.

4. ALIDINA, K. 2013. Professionalism in postlicensure nurses in developed countries. Journal of Nursing Education and Practice, 3, 128.

5. Mueller, P. S. (2015). Teaching and assessing professionalism in medical learners and practicing physicians. Rambam Maimonides medical journal, 6.

6. TERZIOGLU, F., TEMEL, S. \& USLU SAHAN, F. 2016. Factors affecting performance and productivity of nurses: professional attitude, organisational justice, organisational culture and mobbing. Journal of Nursing Management, 24, 735-744.

7. Farhadi, A., Elahi, N., \& Jalali, R. (2016). The effect of professionalism on the professional communication between nurses and physicians: A phenomenological study. Journal of Nursing and Midwifery Sciences, 3, 18-26.

8. Balang, R. V., \& Burton, R. L. (2014). Professionalism: An enduring insight of the nursing profession in Malaysia.

9. Kalisch, B. J., \& Kalisch, P. A. (1983). Improving The. Ajn, American Journal of Nursing, 83, 48-51.

10. BUNKENBORG, G., SAMUELSON, K., ÅKESON, J. \& POULSEN, I. 2013. Impact of professionalism in nursing on in- hospital bedside monitoring practice. Journal of advanced nursing, 69, 1466-1477.

11. Karadağ, A., Hisar, F., Çelik, B., \& Baykara, Z. G. (2016). Determining professionalism in Turkish students nurses. Journal of Human Sciences, 13, 674-682. 
12. Fantahun, A., Demessie, A., Gebrekirstos, K., Zemene, A., \& Yetayeh, G. (2014). A cross sectional study on factors influencing professionalism in nursing among nurses in Mekelle Public Hospitals, North Ethiopia, 2012. BMC nursing, 13, 10.

13. Lombarts, K. M., Plochg, T., Thompson, C. A., Arah, O. A., \& Consortium, D. P. (2014). Measuring professionalism in medicine and nursing: results of a European survey. PloS one, 9.

14. Karadağ, A., Hisar, F., \& Elbaş, N. Ö. (2007). The level of professionalism among nurses in Turkey. Journal of Nursing Scholarship, 39, 371-374.

15. Dikmen, Y., Karataş, H., Arslan, G. G., \& Ak, B. (2016). The level of professionalism of nurses working in a hospital in Turkey. Journal of caring sciences, 5, 95 .

16. Tanaka, M., Taketomi, K., Yonemitsu, Y., \& Kawamoto, R. (2017). The current status of nursing professionalism among nursing faculty in Japan. journal of nursing research, 25, 7-12.

17. Levi, M., \& van der Poll, T. (2010). Inflammation and coagulation. Critical care medicine, 38, S26S34.

18. Saad, L. (2006). Nurses top list of most honest and ethical professions. Gallup News Service, 14, 1-4.

19. Meretoja, R., Isoaho, H., \& Leino- Kilpi, H. (2004). Nurse competence scale: development and psychometric testing. Journal of advanced nursing, 47, 124-133. 